\title{
Effect of the Doping Layer Concentration on Optical Absorption in Si $\delta$-Doped GaAs Layer
}

\author{
Hassen Dakhlaoui \\ Department of Physics, Faculty of Science Bizerte, Bizerte City, Tunsia \\ Email: h_dakhlaoui@yahoo.fr
}

Received June 15, 2012; revised July 19, 2012; accepted July 29, 2012

\begin{abstract}
We study in this paper the intersubband optical absorption of $\mathrm{Si} \delta$-doped GaAs layer for different applied electric fields and donors concentration. The electronic structure has been calculated by solving the Schrödinger and Poisson equations self-consistently. From our results, it is clear that the subband energies and intersubband optical absorption are quite sensitive to the applied electric field. Also our results indicate that the optical absorption depends not only on the electric field but also on the donor's concentration. The results of this work should provide useful guidance for the design of optically pumped quantum well lasers and quantum well infrared photo detectors (QWIPs).
\end{abstract}

Keywords: $\delta$-Doped GaAs; Self-Consistently; The Intersubband Absorption

\section{Introduction}

$\mathrm{Si} \delta$-doped semiconductor structures have been of great interest because of their technological utility in electronic and photonic devices [1,2]. In these structures, a layer of $\mathrm{Si}$ atoms provides electron and gives rise to quantum subbands. By this means, a two-dimensional electron gaz can be obtained by planar doping of GaAs at high donors concentration. Hence there is great interest in a good understanding of $\mathrm{Si} \delta$-doped as a representative example of those devices. Theoretical studies of the above systems usually neglect possible effects of disorder due to the random distribution of impurities in order to simplify the analysis. Indeed, currently available techniques allow for an optimal control of the growing heterostructures, thus justifying the assumption that the ionized impurity atoms are homogeneously distributed inside the $\delta$-doped layers. This approximation has recently been shown to be correct in the high density limit [3]. A number of researches have considered this limit within different approaches, like the Thomas-Fermi [4], local density approximation (LDA) [5] and Hartree methods [6]. These previous works show that in the absence of external fields the Thomas-Fermi semiclassical approach is equivalent to a self-consistent formulation over a wide range of doping concentrations [4]. The effects of applied electric field have recently been considered in the case of single and periodically $\mathrm{Si} \delta$-doped GaAs $[7,8]$ by using a generalized Thomas-Fermi formalism. The electric field dependence of the intersubband optical absorption is also interesting for potential device applications. Intersubband absorption in quantum wells have been proposed or demonstrated experimentally to be very useful for far-infrared detectors [9,10], electro-optical modulators [11,12], and infrared lasers [13]. One of the most remarkable feature of 2DEG is the intersubband optical transitions between the size quantized subbands in the same band. The behavior of an excited quantum well under the influence of an external electric field has been studied before [14, 15]. Also for intersubband absorption, doping is very important to provide the carriers for the ground subband. The intersubband optical absorption in quantum well structures $[16,17]$ and in $\delta$-doped semiconductors has been studied before [18-20].

In the present paper, we investigate theoretically the electronic structure of $\mathrm{Si} \delta$-doped GaAs using a selfconsistent procedure to solve Schrödinger and Poisson equations simultaneously. We have studied the influence of the electric field on the intersubband optical absorption. In addition to the electric field we studied the effect of the donor's concentration on the optical absorption; we conclude that the intersubband transitions are quite sensitive not only to the applied electric field but also to the donor's concentrations.

\section{The Model}

For calculations which describe the structure, the selfconsistent solution of the Schrödinger and Poissson equations in the effective mass approximation was used. The material studied was GaAs with $\mathrm{Si} \delta$-doped layer. The wave function of electrons were decoupled into free par- 
ticule waves in the plane $(x, y)$ and the bound state in the $z$-direction. The structure was modelled assuming uniform distribution of the donors in the $\delta$-doped layer. Free electrons are captured in the neighbourhood of parent ions by electrostatic interaction. The result of this interaction is the formation of quasi-two-dimensional electron gas around the dopant slab where electrons are free to move in the planes of doping and their motion is bound and quantized in the perpendicular direction. We assume the validity of the effective-mass approximation and take an isotropic and parabolic conduction band in the growth direction. This approximation usually works fine in GaAs. In the envelope function approach, the electronic wavefunction corresponding to the $j$ th subband may be factorized as follows [21]:

$$
\psi_{j}(r)=\frac{1}{\sqrt{S}} \exp \left(i k_{\perp} \cdot r_{\perp}\right) \psi_{j}(z)
$$

where $k_{\perp}$ and $r_{\perp}$ are the in-plane wave vector and spatial coordinates, respectively. Here $S$ is the area of the layer. The subband energy follows the parabolic dispersion law $E_{j}+\hbar^{2} k_{\perp}^{2} / 2 m^{*}, m^{*}$ being the electron effecttive mass at the bottom of the conduction band $(\Gamma$ valley). The quantized energy levels $E_{j}$ and their corresponding envelope functions $\psi_{j}(z)$ satisfy the following Schrodinguer-like equation:

$$
\left(-\frac{\hbar^{2}}{2 m^{*}} \frac{\mathrm{d}^{2}}{\mathrm{~d} z^{2}}+v(z)\right) \psi_{j}(z)=E_{j} \psi_{j}(z) .
$$

The one electron potential splits into three different contributions:

$$
V(z)=V_{H}(z)+V x c(z)+e F z .
$$

The last term in equation is the potential of an external electrical field. The Hartree potential is a result of the electrostatic interaction of electrons with themselves and with ionized dopants. It can be found by solving the one dimensional Poisson-equation,

$$
\frac{\mathrm{d}^{2} V_{H}(z)}{\mathrm{d} z^{2}}=\frac{e}{\varepsilon_{0} \varepsilon_{k}}\left[N_{D}-n(z)\right]
$$

where the elecron density $n(z)$ is given as

$$
n(z)=\sum_{J} n_{j}\left|\psi_{j}(z)\right|^{2} .
$$

And the sum goes over the sub-bands. The number of electrons per unit area in the $j$ th sub-band can be calculated as

$$
n_{j}=\frac{m^{*} K_{B} T}{\pi \hbar^{2}} \ln \left[1+\exp \left(\frac{E_{F}-E_{j}}{K_{B} T}\right)\right],
$$

where $E_{F}$ is the Fermi energy. The exchange and correlation potential $V_{x c}$ was found according to the Hedin and Lundquist parametrization,

$$
V_{x c}(z)=-\frac{R_{y}^{*}}{10.5 \pi \alpha r}\left[1+0.773 r \ln \left(1+r^{-1}\right)\right]
$$

where $\alpha=(4 / 9 \pi)^{1 / 3}, r=\frac{1}{21}\left[\frac{4}{3} \pi a^{*} n(z)\right]^{-1 / 3}$, and $R_{y}=e^{2} / 8 \pi \varepsilon_{0} \kappa a^{*} \approx 5 m e V$ is the effective Rydberg constant and $a^{*}=4 \pi \varepsilon_{0} \kappa \hbar^{2} / m^{*} e^{2} \approx 100 \AA$ is the effective Bohr radius. The solutions of these equations give us the sub-band energy levels, the effective confining potential and also the charge density profiles. The calculation also yields self-consistently the position of the Fermi level $E_{f}$, from the condition that the total number of electrons must equal the total number of donors, i.e. $n_{i}=N_{D}$. All donors are assumed to be ionized and are replaced by uniform distribution. We have solved the Equations (2)-(7) self consistently using the finite difference technique. After the subband energies and their corresponding wave functions is obtained, the linear absorption coefficient $\sum_{i} \alpha(\omega)$ for the intersubband transitions can be clearly calculated as,

$$
\begin{aligned}
\alpha(\omega)= & \frac{\omega \mu c e^{2}}{n_{r}}\left|M_{f i}\right|^{2} \frac{m^{*} K_{B} T}{L_{\text {eff }} \pi \hbar^{2}} \\
& \times \ln \left\{\frac{1+\exp \left[\left(E_{F}-E_{i}\right) / K_{B} T\right]}{1+\exp \left[\left(E_{F}-E_{f}\right) / K_{B} T\right]}\right\} \\
& \times \frac{\hbar / \tau_{i n}}{\left(E_{F}-E_{i}-\hbar \omega\right)^{2}+\left(\hbar / \tau_{i n}\right)^{2}}
\end{aligned}
$$

with the matrix element

$$
M_{f i}=\int_{-L / 2}^{L / 2} \psi_{f}^{*}(z) z \psi_{i}(z) \mathrm{d} z,
$$

where $E_{f i}=E_{f}-E_{i}, E_{f}$ and $E_{i}$ denote the quantized energy levels for the final and initial states, respectively, $\mu$ is the permeability, $C$ is the speed of light in free space, $L_{\text {eff }}$ is the effective spatial extent of electrons in subbands, $n_{r}$ is the refractive index, $\tau_{i n}$ is the intrasubband relaxation time (where $\tau_{\text {in }}$ is a constant and used the numerical value of $0.14 \mathrm{ps}$ following Ref. [14]).

\section{Results and Discussion}

\subsection{Effect of Electric Field on Optical Absorption}

In our numerical simulations, the thickness of GaAs structure is $L=50 \mathrm{~nm}$. The $\delta$-doped layer $(2 \mathrm{~nm})$ is assumed to be inserted into an infinite quantum well. The calculations were done for the temperature $T=295 \mathrm{~K}$. The boundary conditions are $\psi(0)=\psi(L)=0$. The effective 
mass of the electron has been taken as $\left(m^{*}=0.067 m_{0}\right)$, $m_{0}$ being the free electron mass. Figures 1(a)-(c) show us the absorption coefficient as a function of the photon energy for different electric field intensities. It is clear from Figure 1(a) that in absence of electric field one notices the existence only of the following transitions: (1-2); $(2-3)$; (3-4) and one can easily see that the (1-2) intersubband transition is dominant. However this situation changes when the electric field intensity increases. From Figure 1(b) the dominant transition is (3-4) and the maximum of the (1-2) transition decreases. We can note also

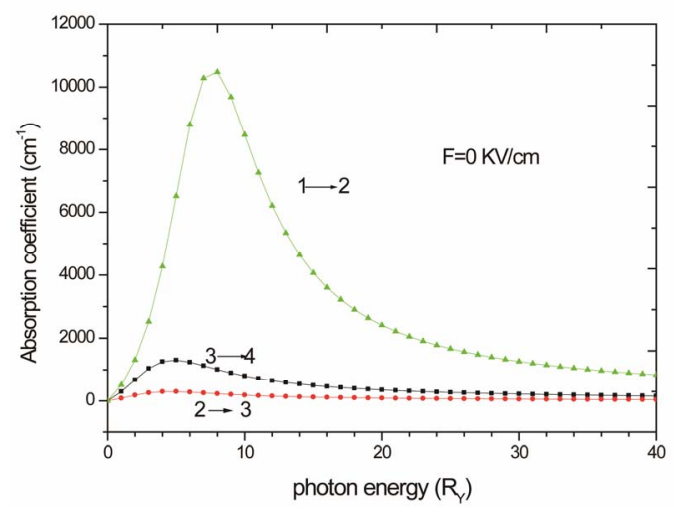

(a)

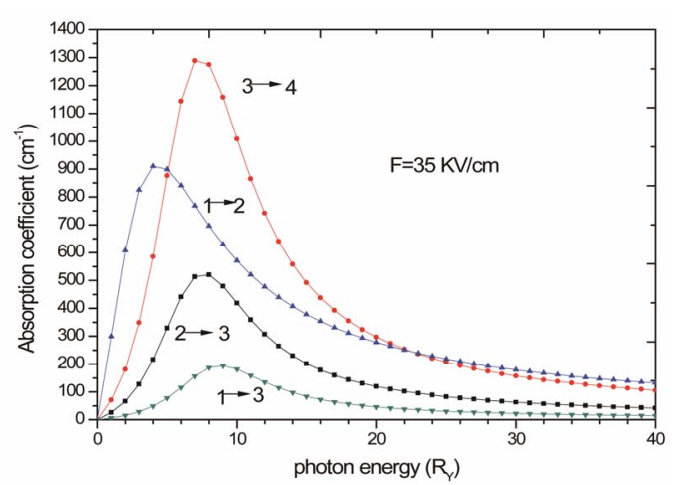

(b)

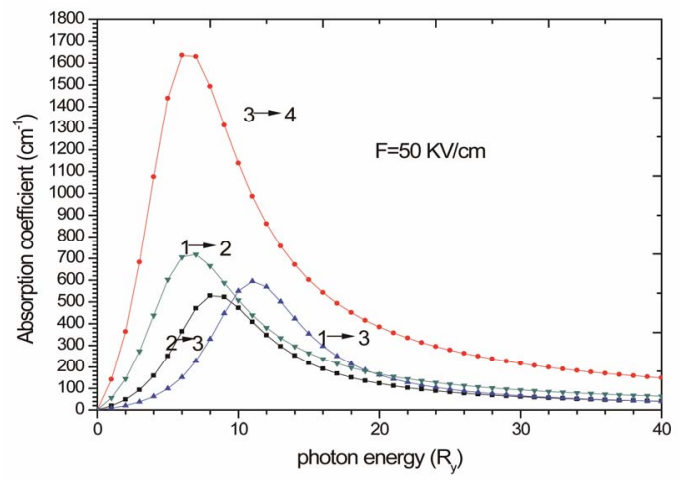

(c)

Figure 1. The variation of the absorption coefficient as a function of the photon energy for different intersubband transitions for (a) $F=0 \mathrm{KV} / \mathrm{cm}$; (b) $F=35 \mathrm{KV} / \mathrm{cm}$; (c) $F=50 \mathrm{KV} / \mathrm{cm}$. that the forbidden transition (1-3) when $\mathrm{F}=0 \mathrm{KV} / \mathrm{cm}$ becomes possible when $\mathrm{F}=35 \mathrm{KV} / \mathrm{cm}$. Figure 1(c) show us that more and more the electric field intensity increases more that the transitions (3-4), (1-3) and (2-3) increases whereas the transition (1-2) decreases. The results stated above can be explained as follows: In absence of an applied electric field the confining potential presents $V$-shape profile and the envelope wavefunctions are symmetric around the doped layer, in this case the transition (1-3) is forbidden. However when the electric field intensity increases the confining potential becomes slightly asymmetric, in other words the confining potential change its shape and a secondary quantum well appears at the left (Figure 2) [22,23]. The excited subbands energies decrease because the asymmetric structures, the higher subband energies, which are more energetic, can easily, penetrate into the secondary quantum well and, thus their energies decrease.

Figure 3 shows the absorption coefficient (1-2) for different applied electric field intensities. It is clear from this figure that when the electric field intensity increases the maximum of absorption decreases, this decrease in

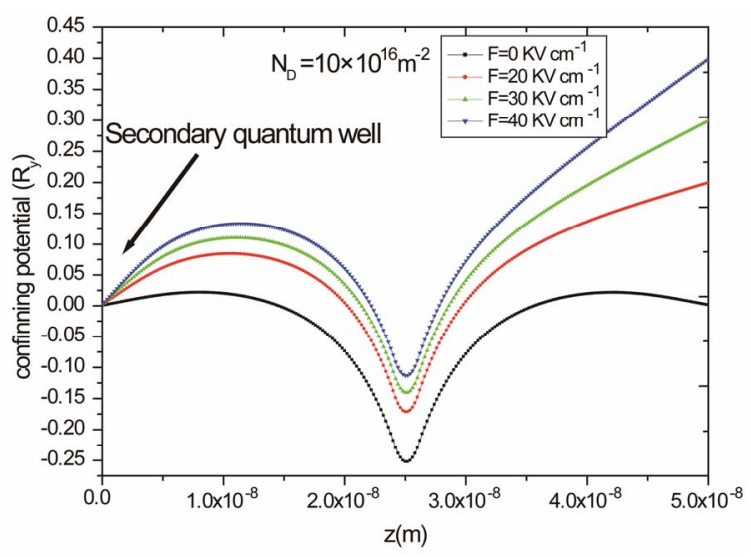

Figure 2. The self consistent confining potential for different electric field intensities.

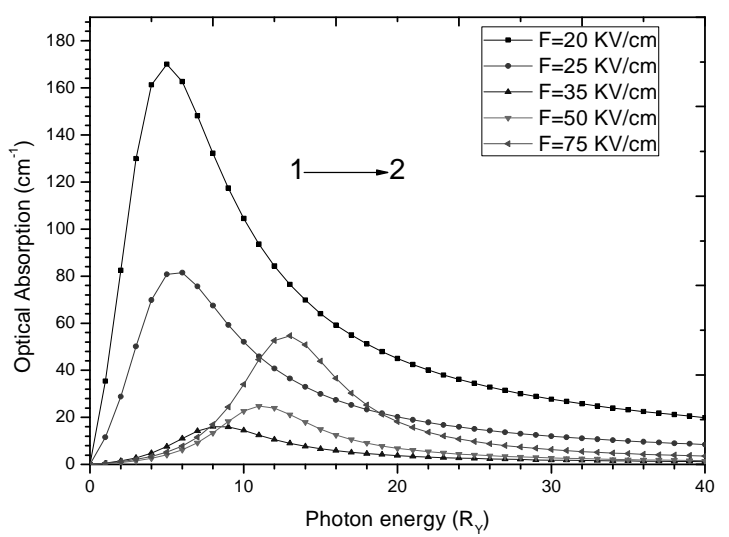

Figure 3. The absorption coefficient of (1-2) intersubband transition for different applied electric field intensities. 
absorption is observed for small applied electric field intensity $\mathrm{F}<35 \mathrm{KV} / \mathrm{cm}$. However for high applied electric field $\mathrm{F}>35 \mathrm{KV} / \mathrm{cm}$ this maximun in absorption coefficient increases with increasing the intensity of applied electric field.

\subsection{Effect of Doping Layer Concentration on Optical Absorption}

To illustrate the effect of the concentration on the properties of our structure one starts with representing the confining potential for various doping layer concentration $N_{D}$ (Figure 4). With increasing the value of the concentration $N_{D}$ the potential becomes more and more deep what improves the confinement of the electrons. We have also study the effect of the concentration $N_{D}$ on the absorption coefficient of the (1-2) intersubband transition (Figure 5). As seen from this figure, while for small concentration range $\left(N_{D}<3 \times 10^{16} \mathrm{~m}^{-2}\right)$ the absorption peak increases with increasing the value of $N_{D}$, however for high concentration rang $N_{D}>3 \times 10^{16} \mathrm{~m}^{-2}$ this peak decreases in magnitude with increasing electric field. Because the high $N_{D}$ concentration generate a deeper potential of confinement (Figure 4) and therefore the levels of energies will be nearer one of the other what decreases the absorption coefficient. Therefore one can say that the concentration of the doping layer can serve to adjust the value of the desired absorption coefficient.

In Figure 6 we plot the energy difference between the ground and the first excited subband, $\Delta E=E_{2}-E_{1}$ as a function of the doping layer concentration $N_{D}$, from this figure we note that this difference between the two ener- gies decreases for $N_{D}<3 \times 10^{16} \mathrm{~m}^{-2}$ range and increases for the $N_{D}>3 \times 10^{16} \mathrm{~m}^{-2}$. This result confirms the value of this concentration limits $N_{D}$ (limit) $=3 \times 10^{16} \mathrm{~m}^{-2}$ found in the previous paragraph (Figure 5). The results open the possibility to design devices for use as optical filters controlled by doping layer concentration.

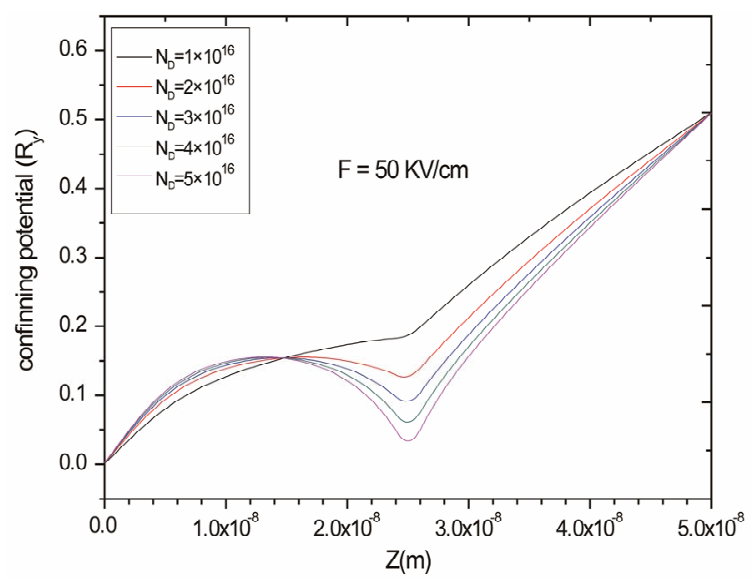

Figure 4. Confining potential for various doping layer concentration $N_{D}$.

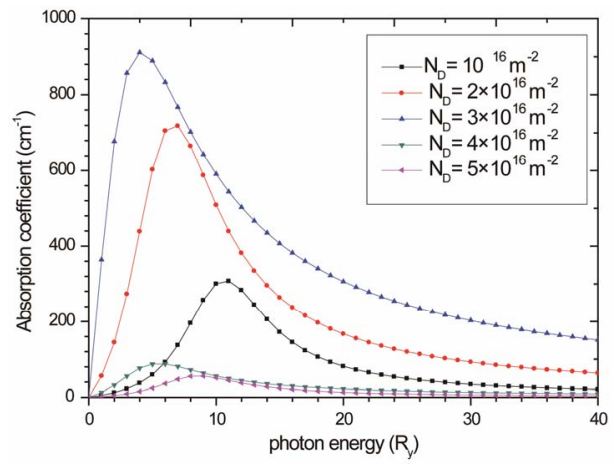

Figure 5. The absorption coefficient of (1-2) intersubband transition for different concentration $N_{D}$ of the doped layer.

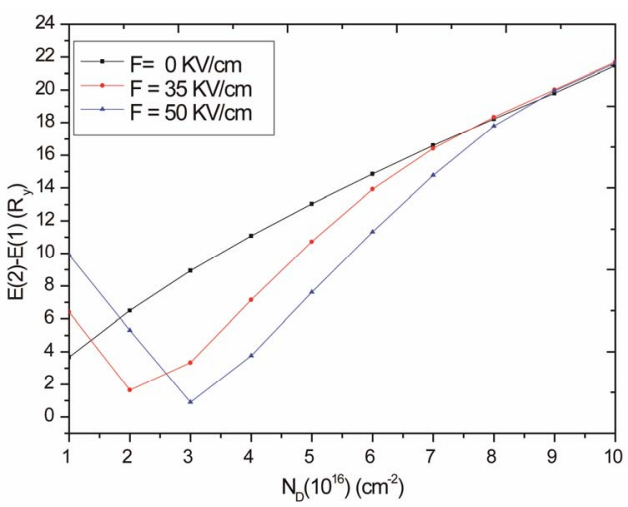

Figure 6. Energy difference between the ground and the first excited as a function of the doping layer concentration.

\section{Conclusion}

We have investigated the intersubband optical absorption of Si $\delta$-doped GaAs for different applied electric fields and various concentrations of the doping layer using a self-consistent procedure to solve the Schrödinger and Poisson equations. From our results, it is clear that the subband energies and intersubband optical absorption is quite sensitive to the applied electric field. Also our results indicate that the optical absorption depends not only on the electric field but also on the doping layer concentration. Such systems are extremely attractive from the point view of both basic research and technological application, such as, optical modulators, high-power FETs, and infrared devices based on the intersubband transition of electrons.

\section{REFERENCES}

[1] E. F. Schubert, A. Fischer and K. Ploog, "The DeltaDoped Field-Effect Transistor," IEEE Transactions on Electron Devices, Vol. 33, No. 5, 1986, pp. 625-632. doi:10.1109/T-ED.1986.22543

[2] K. Ploog, M. Hauser and A. Fischer, "Fundamental Studies and Device Application of $\delta$-Doping in GaAs Layers and in $\mathrm{Al}_{x} \mathrm{Ga}_{1-x} \mathrm{As} / \mathrm{GaAs}$ Heterostructures," Applied Phy- 
sics A: Materials Science \& Processing, Vol. 45, No. 3, 1988, pp. 233-244. doi:10.1007/BF00615010

[3] J. Kortus and J. Monecke, "Formation of Subbands in $\delta$-Doped Semiconductors," Physical Review B, Vol. 49, No. 24, 1994, pp. 17216-17223.

[4] L.Ioratti, "Thomas-Fermi Theory of $\delta$-Doped Semiconductor Structures: Exact Analytical Results in the High Density Limit," Physical Review B, Vol. 41, No. 12, 1990, pp. 8340-8344.

[5] M. H. Degani, "Energy Electron Levels in a $\delta$-Doped Layer in GaAs," Physical Review B, Vol. 44, No. 11, 1991, pp. 5580-5584.

[6] A. Zrenner, F. Koch and K. Ploog, "Subband Physics for a 'Realistic' $\delta$-Doping Layer," Surface Science, Vol. 196, No. 1-3, 1988, pp. 671-676.

[7] F. Dominguez-Adame, B. Mendez and E. Macia, "Electronic Structure of Si Delta-Doped GaAs in an Electric Field," Semiconductor Science and Technology, Vol. 9, No. 3, 1991, p. 263.

[8] F. Dominguez-Adame and B. Mendez, "Stark Ladders in Periodically Si- $\delta$-Doped GaAs," Physical Review B, Vol. 49, No. 16, 1994, p. 11471.

[9] X. Zheng, T. K. Carns, K. L. Wang and B. Wu, "Electron Mobility Enhancement from Coupled Wells in DeltaDoped GaAs," Applied Physics Letters, Vol. 62, No. 5, 1993, pp. 504-507. doi:10.1063/1.108893

[10] R. J. Turton and M. Jaros, "Intersubband Optical Transitions in $\mathrm{Si}_{-} \mathrm{Si}_{0.5} \mathrm{Ge}_{0.5}$," Applied Physics Letters, Vol. 54, No. 20, 1989, pp. 1986-1988. doi:10.1063/1.101190

[11] D. Ahn and S. L. Chuang, "Intersubband Optical Absorption in Quantum Well with an Applied Electric Field," Physical Review B, Vol. 35, No. 8, 1987, p. 4149. doi:10.1103/PhysRevB.35.4149

[12] D. Ahn and S. L. Chuang, "Exact Calculations of Quasibound States of an Isolated Quantum Well with Uniform Electric Field: Quantum-Well Stark Resonance," Physical Review B, Vol. 34, No. 12, 1986, pp. 9034-9037. doi:10.1103/PhysRevB.34.9034

[13] R. F. Kazarinov and R. A. Suris, "Possibility of the Amplification of Electromagnetic Waves in a Semiconductor with a Superlattice," Soviet Physics-Semiconductors, Vol. 5, No. 4, 1971, p. 707.
[14] D. Ahn and S. L. Chuang, "Optical Transitions in a Parabolic Quantum Well with an Applied Electric Field-Analytical Solutions," IEEE Journal of Quantum Electronics, Vol. 23, No. 12, 1987, p. 2196.

[15] C. T. Giner and J. L. Gondar, "Exact Wave Functions and Energy Levels for a Quantum Well with an Applied Electric Field," Physica $A$ \& B, Vol. 138, No. 3, 1986, pp. 287-294. doi:10.1016/0378-4363(86)90009-4

[16] N. G. Semaltianos, "Photoluminescence Studies of GaAs/ AlGaAs Multiple Quantum Well Heterostructures," Journal of Physics and Chemistry of Solids, Vol. 63, No. 2, 2002 , pp. 273-277. doi:10.1016/S0022-3697(01)00140-8

[17] V. Albe and L. J. Lewis, "Optical properties of InAs/InP Ultrathin Quantum Wells," Physica B: Condensed Matter, Vol. 301, No. 3-4, 2001, pp. 233-238. doi:10.1016/S0921-4526(01)00269-1

[18] D. Ahn, "Intersubband Transitions in Doped Semiconductor with an Applied Electric Field: Exact Solutions," Physical Review B, Vol. 48, No. 11, 1993, pp. 7981-7985. doi:10.1103/PhysRevB.48.7981

[19] V. A. Kul'Bachinskii, et al., "Transport and Optical Properties of Tin $\delta$-Doped GaAs Structures," Semiconductors, Vol. 33, No. 7, 1999, pp. 771-778.

[20] K. T. Kim, et al., "Inter-Miniband Optical Absorption in a Modulation-Doped $\mathrm{Al}_{x} \mathrm{Ga}_{(1-x)} \mathrm{As} / \mathrm{GaAs}$ Superlattice," Journal of Applied Physics, Vol. 69, No. 9, 1991, pp. 66176624. doi:10.1063/1.348875

[21] J. A. Cuesta, A. Sanchez and F. Dominguez-Adame, "Self-Consistent Analysis of Electric Field Effects on Si Delta-Doped GaAs," Semiconductor Science and Technology, Vol. 10, No. 10, 1995, p. 1303. doi:10.1088/0268-1242/10/10/002

[22] E. Ozturk and I. Sokmen, "The Electric Field Effects on Intersubband Optical Absorption of $\mathrm{Si} \delta$-Doped Gaas Layer," Solid State Communications, Vol. 126, No. 11, 2003, pp. 605-609. doi:10.1016/S0038-1098(03)00301-6

[23] J. Osvald, "Electronic Properties of a near Surface Si $\delta$-Doped GaAs under an Applied Electric Field," Journal of Physics D: Applied Physics, Vol. 37, No. 19, 2004, p. 2655. doi:10.1088/0022-3727/37/19/007 